\title{
Auch Raucher sind als Spender wichtig
}

\author{
Spenderlungen von Rauchern sind in einem funktionell reduzier- \\ ten Zustand. Das beeinträchtigt den Erfolg der Transplantation. \\ Trotzdem kann auf Spenderlungen von Rauchern nicht \\ verzichtet werden, denn sie ermöglichen kürzere Wartezeiten \\ bis zur Transplantation und damit eine wesentliche \\ Verbesserung der Prognose. Dies macht die retrospektive \\ Datenanalyse von R. S. Bonser et al. deutlich. \\ Lancet 2012; 380: 747-755
}

Die Transplantation von Spenderlungen von Rauchern wird in der Literatur kritisch bewertet. Deren Vorschädigung beeinträchtigt nachhaltig den Transplantationserfolg. Unklar war bisher, in welchem Umfang die Prognose beeinflusst wird. Dabei ist von besonderem Interesse, welchen prognostischen Effekt der Verzicht auf Rauchertransplantate hätte, da die Betroffenen dann dem Risiko ausgesetzt sind auf der Warteliste frühzeitig zu versterben.

Retrospektiv analysierten die Forscher die Daten von 1295 Lungentransplantationen im Zeitraum von 1999-2010 (UK Transplant Registry). 510 Transplantate stammten von Rauchern. Primäres Zielkiterium war die 3-Jahres-Überlebenszeit nach der Transplantation. Zusätzlich wurde geprüft, wie Ko-Risiken die Mortalitätsprognose beeinflussen. Das funktionelle Ergebnis der Transplantation wurde zusätzlich ausgewertet. Die 3-Jahres-Überlebensrate lag bei $67 \%$, wenn das Transplantat von einem status) dagegen nur ca. $65 \%$. identifizieren.
Nichtraucher, bei 56\%, wenn es von einem Raucher stammte. Somit stieg durch den Einsatz des Rauchertransplantats das Risiko um 36\% (adj. HR). Gegenüber dem Verbleib auf der Warteliste war dies trotzdem von Vorteil, denn das Abwarten einer Nichtraucherlunge hätte das 3-JahresMortalitätsrisiko um 21\% (nicht adj. HR) über das Risiko eines Rauchertransplantats hinaus erhöht. Bei der prognostisch besonders schlechten Indikation zystische Fibrose wären somit auf der Warteliste etwa 95\% in der 3-Jahresfrist verstorben, transplantiert (ohne Berücksichtigung Raucher-

Funktionell zeigte das Nichtrauchertransplantat bessere Resultate. Die maximale $\mathrm{FEV}_{1}$ lag bei 2,71 l/s (Raucherlungen 2,42 1/s). Zusätzlich konnten die Forscher den Zytomegaliestatus, die Körpergröße, das Geschlecht und das Alter als wesentliche unabhängige Risiken für die Mortalität

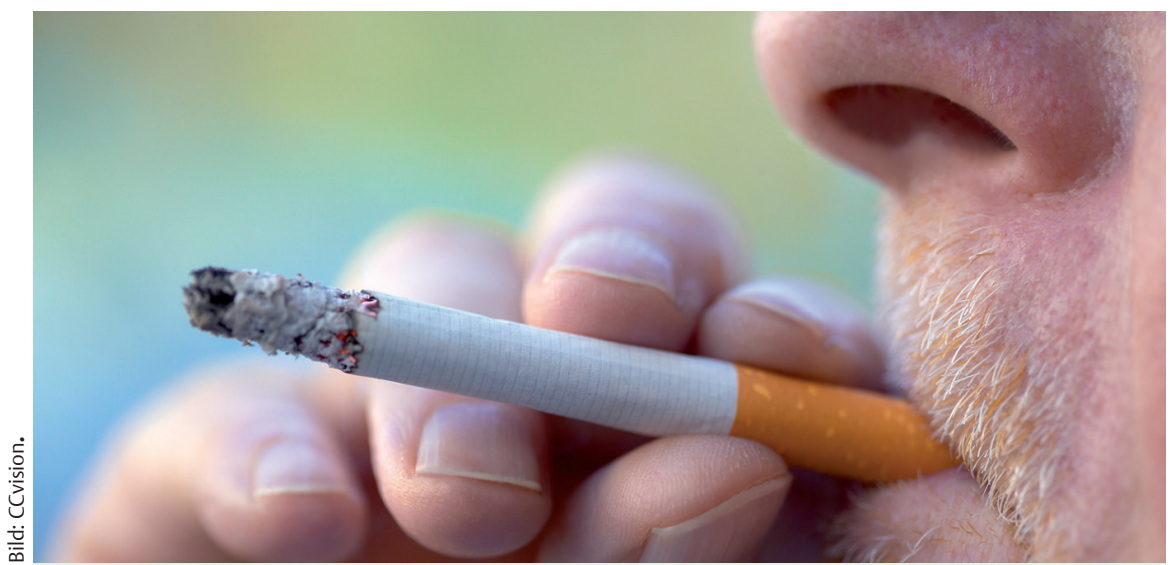

Die Transplantation einer Raucherlunge ist zwar mit einer geringeren 3-Jahres-Überlebensrate verbunden, zeigte jedoch einen Überlebenvorteil gegenüber der Warteliste für eine Nichtraucherlunge.

\section{Fazit}

Der Ausschluss von Rauchern als potenzielle Organspender ist nicht gerechtfertigt, weil dies die Überlebenschancen der Betroffenen wesentlich verschlechtert. Die Autoren geben zu bedenken, dass in der Praxis ca. $40 \%$ der Spenderlungen von Rauchern stammen. Die sich hieraus ergebende Problematik muss deshalb offensiver mit den potenziellen Empfängern besprochen werden.

Dr. Horst Gross, Berlin

Forschung

\section{PulmoStents gegen verengte Bronchien}

Die Textilforschung liefert zusehends Lösungen für den Medizin- und Gesundheitssektor. Nach Fettgewebe-Ersatz, textiler Nervenleitschiene und Therapiehandschuh wird jetzt in Aachen ein Transplantat für Lungenkrebs-Patienten entwickelt: ein mit patienteneigenen Zellen besiedelter Stent mit Selbstreinigungsfunktion speziell für die Bronchien. Er soll die klinisch bereits zur Anwendung kommenden passiven Bronchien-Stents ablösen, die keinen Schutz vor Atemwegsschleim und dem erneuten Einwachsen des Tumors bietet.

Projektleiter Prof. Stefan Jockenhövel von der RWTH Aachen nennt das bis zum Jahr 2016 laufende europäische PulmoStentProjekt mit Experten aus 5 Ländern „bahnbrechend“. Die bereits im Labormuster vorliegende textile Gefäßstütze, der sogenannte PulmoStent, soll aber „frühestens am Ende des Jahrzehnts“ für den klinischen Einsatz zur Verfügung stehen.

Trotz eines vom benachbarten Universitätsklinikum geplanten Tierversuchs mit einem Schaf noch Zukunftsmusik: Das bis zu 4x 1,5 cm große 3D-Metall-Geflecht mit Flimmerhärchen im Inneren, die für den Abtransport von gefährlichen Verschleimungen sorgen, soll dann ähnlich wie bei den seit Jahren gebräuchlichen Gefäßstents bei Bypassoperationen am Herzen in die menschlichen Bronchien hinein implantiert werden. Auf diese Weise, so die Forscher, soll sich die durch Lungenkrebs verursachte Verengung der Atemwege ebenso aufhalten lassen wie die Neubildung von Tumoren.

Ronny Eckert, Berlin 